\title{
Antony J. Williams, Gary E. Martin and David Rovnyak (Eds): Modern NMR Approaches to the Structure Elucidation of Natural Products, Volume 1: Instrumentation and Software
}

\author{
Philip J. Sidebottom ${ }^{1}$
}

(C) Springer-Verlag Berlin Heidelberg 2017

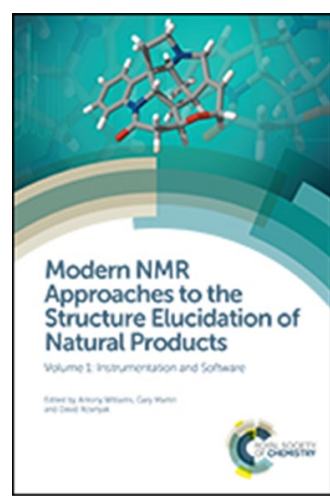

\section{Bibliography}

Modern NMR Approaches to the Structure Elucidation of Natural Products, Volume 1: Instrumentation and Software.

Antony J. Williams, Gary E. Martin, David Rovnyak (Eds)

Royal Society of Chemistry, Cambridge, UK, 2016, pp xii +329

ISBN: 978-1-84973-383-0

GBP 139.99

It is tempting to assume from the title that this book will only contain material of interest to those engaged in the structure elucidation of natural products. In my opinion such an assumption would be a mistake. Those working in other fields where small molecule structure elucidation or the analysis of organic mixtures is necessary, should also find something in this book to interest them. The chapters on magnets and probes are relevant to most NMR spectroscopists.

The stated aim of the editors is "to expose the reader to stateof-the-art technologies in hardware, software and methods". They expect "that many of these will become more commonplace in the near future" and "forewarned is forearmed". Thus, for most readers they hope to provide a look into the future. To do it, in this, the first of a planned two volume series, they have put together 13 chapters written by experts in their fields. These are split more or less evenly between hardware and software and in most cases contain an extensive list of literature

Philip J. Sidebottom

chromatographia@springer.com

1 Stevenage, Hertfordshire, UK references. The book is well produced and in many places the clarity is enhanced by the effective use of colour.

As is the nature of such multi-author works there is overlap between some of the chapters and the style and quality vary between them. The gestation period for this book has been 4 years. As a result, some chapters, presumably those whose authors submitted their material on time, are not quite as up to date as others.

In any multi-topic book each reader will take something different from it. Here are some of my highlights and impressions. In the hidden gem category is the section covering some of the sample preparation issues encountered when only small amounts of analyte are available. This is tucked into the chapter on microprobes. The advances in and power of using NMR features for dereplication as outlined in Chapter 8 also caught my eye. The chapter on computer-assisted structure elucidation seemed at times like an extended advertisement for one particular commercial package. That said, it is clear that much has been achieved in this area. I am left with the impression of a tool that, after appropriate training in its use, will provide help to experts rather than being a replacement for them.

Finally, there is a chapter that from its title appears to be in the wrong book. What is a chapter on atomic force microscopy doing in an NMR book? However, had I skipped this chapter I would have missed one of the best, concise accounts of the structure elucidation process using NMR that I have come across. This culminates by highlighting the difficulties NMR has in solving the structures of molecules where the ratio of protons to heavy atoms is low. It then rightly makes the point that in such cases you may need to consider using other methods. With this in mind it would have been nice to see some coverage of the crystalline sponge approach to X-ray crystallography pioneered by Makoto Fujita's group. Of course this might be the surprise held back for volume 2-we will just have to wait and see. 